\title{
CONFIGURAÇÕES ESTRATÉGICAS E DESEMPENHO ORGANIZACIONAL: em busca de novos imperativos
}

\author{
Strategic configurations and organizational performance: \\ in search for new imperatives
}

\section{Cristiano de Oliveira Maciel ${ }^{[\mathrm{a}]}$, Mauricio Reinert $^{[\mathrm{b}]}$, Camila Camargo ${ }^{[\mathrm{c}]}$}

[a] Doutorando em Administração pela Pontifícia Universidade Católica do Paraná (PUCPR), Curitiba, PR - Brasil, e-mail: crmaciel. adm@gmail.com

[b] Doutor em Administração pela EAESP-FGV, professor adjunto da Universidade Estadual de Maringá (UEM), PR - Brasil, e-mail: m.reinert@uol.com.br

[c] Doutoranda em Administração pela Pontifícia Universidade Católica do Paraná (PUCPR), Curitiba, PR - Brasil, e-mail: caca. adm@gmail.com

\section{Resumo}

O desenvolvimento de configurações estratégicas frequentemente tem se dado a partir de imperativos como ambiente, estrutura, liderança e estratégia. Entretanto, ao considerar a realidade de organizações de pequeno porte, é provável que se encontrem ambientes e, sobretudo, estruturas e estratégias bastante semelhantes num mesmo setor. Desse modo, o presente trabalho se propõe a identificar configurações estratégicas a partir de novos imperativos, como comportamento empreendedor, locus de controle e capacidades organizacionais - variáveis com maior potencial de diferenciação entre organizações de pequeno porte e de explicação da heterogeneidade de desempenho. A partir de um survey que envolveu 130 empresas do ramo de vestuário de Curitiba, PR, e posterior Análise Fatorial Exploratória para validação das escalas, foi identificada, com apoio da Análise de Cluster, uma taxonomia de três grupos de organizações que apresentaram diferenças estatisticamente significativas entre os novos imperativos propostos. Foi também testada a hipótese de que essa taxonomia das configurações explica as diferenças de desempenho, que foi corroborada. Esses resultados indicam que essa taxonomia pode ser útil para a compreensão de configurações estratégicas em empresas de pequeno porte.

Palavras-chave: Configurações. Desempenho. Organizações de pequeno porte.

\section{Abstract}

The development of strategic configurations has frequently occurred from imperatives such as environment, structure, leadership and strategy. However, in considering the reality of small sized organizations, it is probable to find environments as well as structures and strategies in particular that are very similar in one particular 
sector. Therefore, this study aims to identify strategic configurations by studying new imperatives, in this case, entrepreneurial behavior, locus of control and organizational capabilities -variables with a higher differentiation potential in small-sized organizations as well with for better explaining the heterogeneous performance results. Taking into account a survey that included 130 companies that dealt with the clothing business in the city of Curitiba, PR, and with the help of a Cluster Analysis, using an exploratory factorial analysis to validate the scales, it was identified a taxonomy of three organizational groups, presenting statistically significant differences, among the new proposed imperatives. It was tested the hypothesis that this configurations taxonomy explains the differences in their performance, and this hypothesis was validated. These results indicate that this type of taxonomy may be helpful in understanding strategic configurations in small-sized businesses.

Keywords: Configurations. Performance. Small-sized organizations.

\section{INTRODUÇÃO}

A Escola da Configuração, apresentada por Mintzberg, Ahlstrand e Lampel (1998), com origem nos trabalhos de Miller $(1986,1987,1996)$ e de Miller e Friesen (1978), estabelece que a evolução das organizações seja marcada por uma dinâmica quântica entre períodos de estabilidade e períodos mais breves de transformação.

Assim, em períodos de continuidade, são identificados padrões de relacionamentos entre atributos organizacionais com alinhamentos comuns. Entre os imperativos para identificação de gestalts (configurações) estão: ambiente, estrutura, liderança e estratégia. Esses imperativos (MILLER, 1987), que orquestram as interdependências internas de complexos sistemas organizacionais, são apontados como base para geração de tipologias ou taxonomias - classificações geradas empiricamente (MILLER, 1996).

De acordo com Miller (1986, 1987), a exploração dos relacionamentos entre variáveis constituintes das configurações centra na ideia de que tipos comuns organizacionais (gestalts) não podem ser dissociados das características de seu contexto ambiental, nem da exigência de alinhamento entre estrutura, estratégia e liderança. Tais imperativos são os principais responsáveis pela restrição da variedade de configurações, que apresentam congruências naturais internas em determinado conjunto de elementos ambientais e organizacionais.

Todavia, esse parece não ser o caminho mais promissor ao estudar configurações estratégicas no caso de pequenas empresas, sobretudo quando situadas num mesmo ambiente. Organizações de pequeno porte tendem a apresentar estrutura simples e, portanto, mais personalizada, as quais são, com frequência, reflexos de comportamentos e características pessoais do proprietário. Em suas dimensões de formalização e centralização essas estruturas são muito semelhantes - pouco elaboradas (MINTZBERG, 1979). O mesmo pode-se dizer de suas estratégias e capacidade de liderança.

Logo, a pergunta de pesquisa que parece mais adequadamente endereçar a contra-argumentação aos pressupostos de Miller (1986) pode ser expressa do seguinte modo: seria útil elaborar configurações a partir de novos imperativos, organizacionais e comportamentais, para explicação da heterogeneidade de desempenho de organizações de pequeno porte, relegando ambiente, estrutura, estratégia e liderança?

Para tentar responder à questão, foi realizado um levantamento com 130 organizações de pequeno porte em Curitiba, PR. O objetivo do estudo foi identificar uma taxonomia (classificação empírica) de organizações a partir do comportamento empreendedor, locus de controle do proprietário e avaliação das principais capacidades organizacionais, bem como avaliar diferenças de desempenho entre esses grupos em função das configurações.

A escolha desses fatores, como proposta de novos imperativos para identificação de configurações estratégicas, fundamenta-se na influência do comportamento empreendedor em organizações de pequeno porte, na influência do locus de controle sobre tal comportamento e a interdependência do comportamento empreendedor com as capacidades funcionais.

Para consecução do trabalho, após a introdução, o artigo segue com a apresentação do referencial teórico, procedimentos metodológicos, análise dos dados, discussão dos resultados e considerações finais. 


\section{REFERENCIAL TEÓRICO-EMPÍRICO}

\section{Configurações estratégicas}

O termo "configurações estratégicas" (strategic configurations) alcançou notoriedade a partir dos escritos de Danny Miller e Peter H. Friesen, quando estiveram filiados à McGill University e à Ecole des Hautes Etudes Commerciales. A noção de gestalt, oferecida pelos pesquisadores, foi inspirada na ideia de tipos organizacionais comuns, que já vinha sendo explorada no campo dos estudos organizacionais e na área de estratégia (MINTZBERG, 1979; PUGH; HICKSON; HININGS, 1969; PORTER, 1980; MILES; SNOW, 1978).

Sob o rótulo de "configurações" (ou "gestalts", ou "arquétipos", ou "tipos genéricos") (MILLER, 1986, p. 236), essa escola de pensamento em estratégia (MINTZBERG; AHLSTRAND; LAMPEL, 1998) tem por princípio a integração das mensagens de outras perspectivas do campo dentro de um continuum marcado pela estabilidade e pela mudança em seus extremos. Dessa forma, a qualidade dessa abordagem metodológica (MILLER, 1996) possibilita o delineamento de conjuntos de elementos e a exploração de suas complementaridades internas, indo além da abordagem de "uma variável por vez".

O ponto de partida da discussão sobre configurações estratégicas reside no binômio mudança/estabilidade organizacional. Seguindo a lógica de escolas para a categorização de diferentes perspectivas de estudo sobre estratégia, Mintzberg, Ahlstrand e Lampel (1998) afirmam que a Escola da Configuração vê a ação estratégica ora envolvida com a mudança, ora lidando com a continuidade. Juntas, estabilidade e mudança espelham a busca pela eficiência em certos períodos de tempo, e a busca por revitalização e inovação em outros (JARZABKOWSKI, 2004).

Contudo, a Escola da Configuração estaria mais voltada para análise das características organizacionais em períodos de continuidade (MILLER, 1986, 1996), pois na maior parte das vezes a estabilidade de determinado conjunto de características é o atributo mais revelador do que é distinto em dado sistema organizacional. No entanto, os autores dessa escola não relegam a mudança, eles apenas assumem que frequentes períodos de estabilidade são interrompidos por processos ocasionais de transformação (MINTZBERG; AHLSTRAND; LAMPEL, 1998).

Tais processos de mudança podem dar-se por meio de choques eventuais, oscilações, ciclos de vida e mudanças regulares. Apenas a última, a mudança regular, não seria típica de análise nessa escola. Os demais tipos de transformação são concebidos como mudanças quânticas, mudança de vários elementos ao mesmo tempo. Nesses termos, as organizações saltariam de um estado de estabilidade para outro (MINTZBERG; AHLSTRAND; LAMPEL, 1998). Aparentemente, esse é um argumento de exclusão de casos atípicos e conflitantes com as premissas da Escola da Configuração (processos de mudanças incrementais).

Qualquer forma, por conta das alternâncias ocasionais entre um estado e outro, a estabilidade e o ajuste interno de alguns atributos organizacionais são tendências naturais rumo à formação de gestalts, ou, mais precisamente, configurações estratégicas para quem segue essa escola de pensamento nos estudos sobre estratégia (MILLER, 1986, 1996).

Quanto à forma de identificação dessas configurações estratégicas, os autores dessa escola têm buscado adotar duas abordagens principais: tipologias conceituais e taxonomias empíricas. A abordagem de tipologias tem sido empregada para diferenciar organizações entre tipos de estratégias, estruturas e estilos de processo decisório. Ao mesmo tempo em que as tipologias geradas teoricamente são memoráveis (por meio de contrastes facilitam o progresso empírico), elas apresentam como principal fraqueza uma aparente arbitrariedade nos passos seguidos pelo pesquisador para o seu desenvolvimento (MILLER, 1996).

Por outro lado, taxonomias não são geradas de modo tão flexível quanto o desenvolvimento de tipologias. Normalmente, os pesquisadores que seguem essa abordagem buscam identificar grupos naturais (natural clusters) em meio aos dados. O principal mérito da abordagem taxonômica está na validade e significância do conjunto de atributos em que se baseia a classificação de um rol de organizações (MILLER, 1996).

Mais especificamente, é por meio do emprego da segunda abordagem de desenvolvimento de configurações (taxonomias) que se busca, neste trabalho, a identificação de novos imperativos para o delineamento de configurações que possam 
explicar a diferença de desempenho de organizações de pequeno porte.

\section{Novos imperativos para identificação de configurações estratégicas}

Como já colocado, propõe-se aqui a utilização do comportamento empreendedor, do locus de controle do dirigente e capacidades funcionais como novos imperativos para o delineamento de configurações estratégicas de organizações de pequeno porte. Considerar o comportamento empreendedor como variável de gestalts organizacionais comuns significa atentar para a influência de um dos aspectos mais substanciais da filosofia de gerenciamento: a busca pelo crescimento por meio da exploração de oportunidades ambientais (SNOW; MILES; MILES, 2005). Cooper e Dunkelberg (1986) definem a intensidade dessa busca pelo crescimento organizacional a partir da exploração de oportunidades como graus de comportamento empreendedor (degree of entrepreneurship). E assim, focalizam-se principalmente as atividades estratégicas (comportamento) envolvidas na estruturação daquilo que se toma sob o rótulo de empreendedorismo ou comportamento empreendedor.

Nesses termos, o comportamento empreendedor, primeiro imperativo proposto, contempla essencialmente os seguintes elementos: (i) iniciativa, (ii) inovação e (iii) exploração de oportunidades (BIRKINSHAW, 1997; BROWN; DAVIDSSON; WIKLUND, 2001; GALUNIC; RODAN, 1998; JACOBSON, 1992). Sharma e Chrisman (1999, p. 17) reforçam essa constituição do conceito quando afirmam que o empreendedorismo "envolve atos de criação, renovação ou inovação organizacional que ocorrem dentro ou fora de uma organização existente".

Desdobrando o conceito, é possível entender que a iniciativa é definida como um compromisso com o comportamento proativo em relação ao avanço em novas maneiras de uma organização usar ou expandir seus recursos alterando as regras de um setor ou economia (BIRKINSHAW, 1997; GALUNIC; RODAN, 1998). Logo, a iniciativa estaria intrinsecamente ligada à inovação. $\mathrm{O}$ comportamento da firma baseado em oportunidades prima pelo reconhecimento e exploração dessas, independentemente das características da
organização(BROWN;DAVIDSSON; WIKLUND, 2001; SHANE; VENKATARAMAN, 2000; STEVENSON; JARILLO, 1990). Sobretudo, estes elementos - iniciativa, inovação e exploração de oportunidades - estão invariavelmente voltados para um dos principais objetivos do empreendedor: o desempenho estratégico (McGRATH, 2002).

O segundo imperativo proposto é o locus de controle. Associado ao comportamento empreendedor, e até figurando como antecedente de tal comportamento, está o locus de controle do dirigente do negócio. O termo foi inicialmente abordado por J. B. Rotter, em 1954, no livro Social learning and clinical psychology (KAUFMANN; WELSH; BUSHMARIN, 1995; LOOSEMORE; LAM, 2004), mas, aos poucos, o conceito também foi sendo ampliado às áreas de empreendedorismo e outras disciplinas (BOYDSTON; HOPPER; WRIGHT, 2000; KAUFMANN; WELSH; BUSHMARIN, 1995).

Nos estudos sobre empreendedorismo, esse conceito tem sido mensurado a partir de duas dimensões centrais: locus interno e locus externo (BOYDSTON; HOPPER; WRIGHT, 2000; KAUFMANN; WELSH; BUSHMARIN, 1995). Pesquisadores têm definido o construto "locus interno de controle como a crença pessoal em que alguém tem influência sobre seus resultados por meio de suas aptidões, esforços ou habilidades; enquanto locus de controle externo é a crença em que forças externas controlam seus resultados" (KAUFMANN; WELSH; BUSHMARIN, 1995, p. 44). O locusinterno de controle está frequentemente associado de forma positiva com iniciativas, inovações e exploração de oportunidades. Já o externo apresenta associação negativa com esses mesmos elementos comportamentais do empreendedor (MUELLER; THOMAS, 2001).

O terceiro imperativo para as configurações estratégicas - a qualidade das capacidades organizacionais - também tem sua inclusão justificada no estudo em razão da noção de que tais configurações são voltadas para o alcance de um melhor desempenho (FISS, 2007; MILLER, 1986, 1996). Logo, essas configurações teriam de envolver as principais capacidades funcionais de uma organização. Como sustenta a Visão Baseada em Recursos (VBR), o conjunto de recursos controlados por uma organização são os elementos fundamentais para a compreensão da heterogeneidade do desempenho (BARNEY, 1996; RUGMAN; VERBEKE, 2002). 
Sobre a categorização dos recursos organizacionais, Barney (1996) propôs uma divisão suficientemente diversa, mas parcimoniosa: capital financeiro, capital físico, capital humano e capital organizacional. A categoria de capital financeiro é representada por todos os possíveis recursos financeiros, como capital do empreendedor, recursos em caixa e capital de acionistas e bancos. A categoria de capital físico envolve tecnologia física empregada no núcleo operacional da organização, planta da organização, equipamentos, localização geográfica e acesso a insumos. A categoria de capital humano abarca aspectos organizacionais, como treinamento, experiência, julgamento, inteligência, relacionamentos e insights dos membros do corpo diretivo e dos demais níveis hierárquicos. A categoria de capital organizacional tem como exemplo: estrutura formal, planejamento formal e informal, sistemas de coordenação e controle, cultura, reputação, relações informais (BARNEY, 1996).

Entretanto, apesar da utilidade da classificação de Barney (1996), a VBR ainda é criticada por não apresentar classificações mais claras para diferentes recursos e capacidades. O construto competência organizacional tem sido definido como capacidade de coordenação de um conjunto de recursos, atividades e habilidades organizacionais (SANCHEZ, 2003).

A partir dessa lógica, uma competência essencial da organização envolverá várias competências ou capacidades funcionais (excelência em serviços que exige, principalmente, a coordenação das competências funcionais marketing e gestão de pessoas). Deve-se ter em mente que tal coordenação pode envolver distintos níveis de complexidade e essa complexidade será condicionada pelo número de atividades e habilidades a serem coordenadas pela organização em determinadas situações (KING; ZEITHAML, 2001; LEONARD-BARTON, 1992; SANCHEZ, 2003).

\section{Articulando configurações estratégicas e desempenho organizacional}

Neste ponto, é possível promover a articulação entre configurações estratégicas e desempenho organizacional. Essa relação foi inicialmente estabelecida no trabalho de Miller e Friesen (1978). Os autores descreveram dez arquétipos, configurados a partir de diversas variáveis, que conduziriam ao sucesso ou insucesso organizacional. Seis arquétipos teriam maior potencial para conduzir ao sucesso e outros quatro ao fracasso.

Em outro artigo, Miller (1987) afirma que a lógica interna de uma configuração pode produzir variados graus de qualidade dos resultados organizacionais. Configurações estratégicas, como aquelas propostas por Miles e Snow (1978) e Porter (1980), também são associadas a diferentes graus de desempenho em alguns levantamentos:

Em anos recentes o campo de estratégia/ política de negócios tem feito avanços significativos. O trabalho conceitual de Porter (1980) e os estudos empíricos do PIMS realizados por Hambrick e seus colaboradores (1983, 1983a) estão entre os mais interessantes. Estes autores têm derivado tipologias e taxonomias empíricas extremamente interessantes sobre estratégia, focalizando variáveis que têm chamado muita atenção dos economistas industriais - variáveis que mostraram frequentemente influenciarem o desempenho (MILLER, 1986, p. 234).

Com frequência, diferentes configurações ou arquétipos baseados na capacidade de inovação, utilização de recursos, qualidade e amplitude de linha de produtos têm apresentado associações com variáveis de desempenho como retorno sobre o investimento (ROI), crescimento de participação de mercado e variabilidade dos lucros. Por causa das complexas variações de diferentes imperativos em variadas configurações ocorreriam diferenças significativas no desempenho (MILLER, 1986).

Logo, é possível supor que diferentes organizações, com diferentes graus de locus de controle, comportamento empreendedor e capacidades organizacionais acabam por dar forma a diferentes configurações que teriam potencial para explicar a heterogeneidade de desempenho dessas empresas. Daí derivou-se a hipótese do presente estudo, que foi testada em uma amostra de organizações varejistas de pequeno porte na cidade de Curitiba: $\mathrm{H}_{1}$. Em organizações de pequeno porte, diferentes configurações organizacionais baseadas em locus de controle, comportamento empreendedor e capacidades funcionais possuem desempenho diferente. 


\section{PROCEDIMENTOS METODOLÓGICOS}

Olevantamento (survey) foi escolhido como método de coleta de dados no delineamento do estudo. A pesquisa é classificada como exploratória, sem pretensão de apresentar dados conclusivos (BABBIE, 1998). Em relação à dimensão temporal, foi adotada uma perspectiva seccional, ou seja, realização de um levantamento em um ponto no tempo. A unidade de análise é a organização e o nível de análise é organizacional.

A população estudo foi delimitada em todos os varejistas de vestuário da cidade de Curitiba. Todavia, em face de fatores como tempo reduzido e o investimento que seria necessário para a realização de um censo ou amostra probabilística, optou-se pelo procedimento de amostragem não probabilística por conveniência e julgamento.

Os critérios para escolha dos participantes da pesquisa foram os seguintes: setor de atuação (varejo de vestuário), número de funcionários (para representar empresas de pequeno porte) e localização geográfica. A localização geográfica foi fundamental para restringir a amostra aos lojistas que têm suas lojas instaladas na mesma rua que os demais participantes do levantamento. Tentou-se, com isso, minimizar a influência que a localização mais central ou menos central poderia ter sobre o desempenho do empreendimento. A rua em que essas organizações estão instaladas é um local destinado ao comércio e também um ponto turístico onde transitam apenas pedestres e que agrega centenas de lojas do centro da cidade.

O desenvolvimento dos indicadores do questionário se deu após a realização de seis entrevistas individuais com proprietários e gerentes de algumas lojas escolhidas por conveniência. Os respondentes foram inquiridos sobre as competências mais importantes para alcançar um melhor desempenho em seu setor de atuação.

A partir da leitura do material coletado, foram identificados temas (itens de significação) (BARDIN, 1977) que revelaram competências classificadas como capacidades organizacionais funcionais.

Os indicadores da escala de locus de controle interno e externo foram desenvolvidos com base na literatura (BOYDSTON; HOPPER; WRIGHT, 2000; KAUFMANN; WELSH, BUSHMARIN,
1995; LOOSEMORE; LAM, 2004). Os indicadores utilizados na mensuração do comportamento empreendedor foram adaptados de Brown, Davidsson e Wiklund, (2001), Covin e Slevin (1989) e AtuaheneGima e Ko (2001).

Para o desenvolvimento dos itens da escala de desempenho foi utilizado fundamentalmente o apoio da literatura, que apresentou discussões acerca dos consensos e divergências quanto à mensuração do construto, principalmente quanto ao uso de indicadores objetivos e subjetivos (BARNEY, 1996; CANEDO; KRUGLIANSKAS, 1999; CHAKRAVARTHY, 1986; DESS; ROBINSON, 1984; GIMENEZ, 2000; MENNA; ROSSI, 2001; NAMAN; SLEVIN, 1993; VENKATRAMAN; PRESCOTT, 1990).

Os itens finais de natureza subjetiva utilizados na mensuração do desempenho foram:

1) desempenho em relação aos concorrentes;

2) satisfação em relação ao investimento;

3) crescimento das vendas;

4) crescimento/redução das atividades da organização;

5) indicadorinvertido em relação ao item 4;

6) sucesso do negócio em relação aos concorrentes;

7) retorno financeiro;

8) probabilidade de sobrevivência no longo prazo.

Esses indicadores foram avaliados considerando uma amplitude temporal de dois anos. Outras questões foram destinadas e avaliar idade e sexo do proprietário e também tempo no negócio. Cada capacidade organizacional foi avaliada por um indicador em uma escala de cinco pontos (muito fraco a muito forte em relação aos concorrentes ${ }^{1}$ ). Os indicadores de desempenho também foram avaliados em escala de Likert (1 para discordo totalmente a 5 para concordo totalmente), assim como os indicadores dos demais construtos.

Foram também coletados dados dos proprietários, como idade e escolaridade (curso superior ou não), e sobre a empresa, como o tempo de existência em anos. Os dados da variável ensino

1 Essa abordagem de um único indicador para cada competência já foi utilizada por Snow e Hrebiniak (1980). 
superior foram coletados como variável categórica, mas pelo fato de poucos respondentes se incluírem nas categorias segundo grau e ensino superior, optouse por transformar a variável em dummy, possuir ou não ensino superior. Esses dados foram utilizados como variáveis de controle no modelo, visto que características do proprietário, tais como experiência, refletida pela idade, e formação profissional, refletida pela escolaridade, bem como tempo de existência da empresa, podem influenciar o desempenho da organização.

A aplicação do instrumento de coleta de dados resultou em 143 questionários, dos quais 13 foram ignorados por alto número de valores faltantes. Entre os 130 questionários utilizados na análise, ainda existiam alguns com valores faltantes; portanto, fez-se necessário a realização de uma análise desses dados, na qual não foi identificado nenhum padrão, o que possibilita caracterizá-los como faltantes completamente ao acaso (MCAR - missing completely at random) (HAIR, 1995).

A partir da confrontação entre o referencial teórico e os dados coletados, foram identificadas as variáveis que melhor se adaptavam ao modelo a ser testado, mas que também possuíam poucos dados perdidos. Os critérios de parcimônia no número de variáveis independentes (VI) do modelo são importantes por causa da pequena amostra utilizada. Incluir mais algumas variáveis poderia comprometer a significância estatística pelo número de VIs em relação às observações e à diminuição das observações em virtude do aumento dos dados perdidos (PEDHAZUR; SCHMELKIN, 1991;
HAIR, 1995). Para a análise fatorial e de clusters foram incluídos todos os 130 casos. Já no modelo a ser testado foram incluídos apenas 117 casos, por consequência dos valores faltantes nas variáveis das empresas e nas características sociodemográficas dos proprietários.

Os dados foram exportados do Excel para o SPSS 13.0 e submetidos às seguintes análises estatísticas: Análise Fatorial, Análise de Cluster e Modelo Linear Generalizado Univariado. Os resultados das análises constam na seção seguinte do artigo.

\section{ANÁLISE DOS DADOS}

Na primeira parte do tratamento dos dados foram realizadas duas análises fatoriais exploratórias, uma para os indicadores dos imperativos das configurações e outra para os indicadores do desempenho, procurando identificar se as variáveis coletadas se encaixavam nos construtos conforme proposto pela teoria (HAIR et al., 1995). Os resultados das variáveis dos imperativos são apresentados na Tabela 1, e perfizeram conforme o esperado, ou seja, todas as variáveis se ajustaram aos construtos como proposto pela teoria (fator de carregamento próximo de 0,50) (HAIR et al., 1995). Além disso, foi utilizada a técnica do Alfa de Cronbach para avaliar a consistência interna da escala (HAIR et al., 1995). Todos eles apresentaram resultados próximos ou acima de 0,70, o que indica consistência interna satisfatória, possibilitando a utilização dos construtos no modelo a ser testado.

TABELA 1 - AFE para capacidades, comportamento empreendedor, locus de controle

(continua)

\begin{tabular}{|c|c|c|c|c|}
\hline & Capacidades & Locus E. Controle & Comp. Empreendedor & Locus I. controle \\
\hline V01 & 0,496 & & & \\
\hline V02 & 0,470 & & & \\
\hline V03 & 0,521 & & & \\
\hline V04 & 0,658 & & & \\
\hline V05 & 0,791 & & & \\
\hline V06 & 0,751 & & & \\
\hline V07 & 0,724 & & & \\
\hline V08 & 0,608 & & & \\
\hline
\end{tabular}


MACIEL, C. O.; REINERT, M.; CAMARGO, C.

TABELA 1 - AFE para capacidades, comportamento empreendedor, locus de controle (conclusão)

\begin{tabular}{|c|c|c|c|c|}
\hline & Capacidades & Locus E. Controle & Comp. Empreendedor & Locus I. controle \\
\hline V09 & & 0,881 & & \\
\hline V10 & & 0,942 & & \\
\hline V11 & & 0,929 & & \\
\hline V12 & & & & 0,882 \\
\hline V13 & & & & 0,884 \\
\hline V14 & & & 0,724 & \\
\hline V15 & & & 0,649 & \\
\hline V16 & & & 0,796 & \\
\hline V17 & & & 0,567 & \\
\hline Variância & $19,97 \%$ & $15,75 \%$ & $14,25 \%$ & $11,28 \%$ \\
\hline Alfa & 0,813 & 0,915 & 0,681 & 0,744 \\
\hline
\end{tabular}

Nota: KMO: 0,776

Teste de esfericidade qui-quadrado: 785,885 df 105 sig: 0,000

TABELA 2 - AFE não rotacionada para desempenho

\begin{tabular}{lc}
\hline & Desempenho Organizacional \\
\hline V18 & 0,568 \\
V19 & 0,761 \\
V20 & 0,799 \\
V21 & 0,900 \\
V22 & 0,665 \\
V23 & 0,878 \\
V24 & 0,874 \\
V25 & 0,762 \\
\hline Variância & $61,33 \%$ \\
\hline Alfa & 0,906 \\
\hline
\end{tabular}

Nota: KMO: 0,866

Teste de esfericidade qui-quadrado: 675,773 df 28 sig: 0,000

O resultado da análise fatorial dos indicadores de desempenho também correspondeu à teoria e é apresentado na Tabela 2 (fator de carregamento acima de 0,50) (HAIR et al., 1995). O Alfa de Cronbach indica uma consistência interna satisfatória, possibilitando do mesmo modo a utilização das variáveis no construto.

Após a identificação dos fatores, foi realizada uma análise de cluster para a criação de uma taxonomia das configurações a partir dos imperativos propostos no referencial teórico. A análise foi realizada em duas partes. Primeiro, foi feita a análise de cluster hierárquica para a definição do número de clusters e, em seguida, a análise de cluster não hierárquica, pelo método Ward. Foram definidos três grupos. Como na análise dos três clusters o construto Locus Interno de Controle não apresentou diferenças significativas entre as médias de nenhum dos grupos, optou-se por retirá-lo da análise por sua incapacidade de diferenciar as configurações da taxonomia. Foi rodada novamente a análise, utilizando apenas os construtos Capacidades Organizacionais, Locus Externo de Controle e Comportamento Empreendedor. Cada um dos clusters passou a ser chamado de configuração. Os resultados são apresentados na Tabela 3 .

$\mathrm{Na}$ Configuração 1 (C1), foram agrupadas 26 organizações. A sua característica distintiva é apresentar uma média de Locus de Controle Externo bem acima dos outros dois grupos, enquanto a média da Capacidade Organizacional e do Comportamento 
TABELA 3 - Teste Tukey para análise de diferença das médias por configuração

\begin{tabular}{lccc}
\hline & Configuração 1 (C1) & Configuração 2 (C2) & Configuração 3 (C3) \\
\hline Número de Organizações & 26 & 69 & 35 \\
Capacidades Organizacionais & $3,54_{\mathrm{C} 2, \mathrm{C} 3}$ & $3,91_{\mathrm{C} 1, \mathrm{C} 3}$ & $3,25_{\mathrm{C} 1, \mathrm{C} 2}$ \\
Locus Externo de Controle & $3,57_{\mathrm{C} 2 \mathrm{C} 3}$ & $1,67_{\mathrm{C} 1, \mathrm{C} 3}$ & $1,98_{\mathrm{C} 1, \mathrm{C} 2}$ \\
Comportamento Empreendedor & $3,63_{\mathrm{C} 3}$ & $3,86_{\mathrm{C} 3}$ & $3,07_{\mathrm{C} 1, \mathrm{C} 2}$ \\
\hline
\end{tabular}

Nota: A indicação ao lado da média aponta as configurações com médias estatisticamente diferentes a 95\%

Fonte: Dados primários.

Empreendedor está no nível intermediário entre as duas outras configurações.

$\mathrm{Na}$ Configuração 2 (C2), foram agrupadas 69 organizações. O fator distintivo dessa configuração é que ela está sempre nos extremos. As organizações que pertencem a ela têm a mais baixa média de Locus de Controle Externo e as mais altas em Capacidades Organizacionais e em Comportamento Empreendedor.

$\mathrm{Na}$ Configuração 3 (C3), foram agrupadas 35 organizações. O fator distintivo das organizações que pertencem a ela é a menor média em relação a Comportamento Empreendedor e Capacidades Organizacionais, tendo uma média menor que (3) Locus Externo de Controle.

Os resultados indicam que os construtos Capacidades Organizacionais, Locus de Controle Externo e Comportamento Empreendedor podem ser utilizados como imperativos na construção de uma taxonomia de configurações estratégicas de organizações de pequeno porte. Existe uma variação estatisticamente significativa entre os construtos em relação às médias dos imperativos. Cada construto possui características distintivas entre si, que são fundamentais para essas organizações, pois são o que as diferencia, o que é importante em uma taxonomia desse tipo. O fato de uma das configurações contar com mais de $50 \%$ das organizações da amostra pode ser considerado uma limitação, mas não invalida a proposta de taxonomia com base nesses imperativos.

As diferenças entre as configurações são necessárias, mas não suficientes para sustentar essa taxonomia, pois, como discutido no referencial teórico, uma configuração estratégica deve ajudar a explicar as diferenças de desempenho organizacional, que é a hipótese a ser testada por essa pesquisa. Para testá-la, a proposta foi utilizar a técnica estatística GLM Univariado (Generalized Linear Model) presente no pacote estatístico SPSS 13.0. Essa técnica permite testar a hipótese de que as médias de uma Variável Dependente (VD) são diferentes entre grupos (VI). Além disso, diferente da One-Way Anova, é possível acrescentar ao modelo variáveis independentes de controle (VC), categóricas e contínuas, o que permite um melhor refinamento das análises, pois a variância da VD, compartilhada pelas VCs, é retirada antes da variância ser atribuída à VI que está sendo testada (CRAMER, 2006). O modelo a ser testado é apresentado abaixo pela seguinte equação:

$$
\begin{aligned}
\text { Desempenho }= & \mathrm{B}_{0}+\mathrm{B}_{1} \text { Configurações }+ \\
& \mathrm{B}_{2} \text { Ensino Superior }+ \\
& \mathrm{B}_{3} \text { Config*Ens_Sup }+ \\
& \mathrm{B}_{4} \text { Tempo de Existência da } \\
& \text { Empresa }+\mathrm{B}_{5} \text { Idade }
\end{aligned}
$$

Sendo que Desempenho é a VD, o $B_{0}$ é o intercepto, Configurações é a VI a ser testada na hipótese, e as variáveis Ensino Superior, Tempo de Existência da Empresa, Idade do Proprietário e a interação Grupos*Ensino Superior são utilizadas como variáveis de controle.

Para testar o modelo, foi feito inicialmente o teste Levene de igualdade de variância entre os grupos. O teste foi significativo a 99\%. A hipótese de igualdade na variância, portanto, não foi corroborada. Como um dos pressupostos para se testar o modelo 
por meio de GLMé a igualdade de variância entre os grupos, foi necessário fazer um diagnóstico e tomar ações corretivas, pois, apesar de o coeficiente da regressão não ficar enviesado, erro padrão, teste de significância e os intervalos de confiança podem estar incorretos, muito conservadores ou muito sensíveis (COHEN et al., 2003; HAIR, 1995).

Foi identificado que a variância da VD nos grupos está associada aos seus tamanhos: quanto maior o número de indivíduos incluídos, menor a variância. Optou-se, então, pela técnica WLS (Weighted Least Squares), mínimos quadrados ponderados, que foi desenhada para contrabalançar os efeitos adversos da heteroscedasticidade na regressão, pois ela produz o menor erro padrão possível, quando utilizados os pesos adequados na ponderação (PEDHAZUR; SCHMELKIN, 1991; COHEN etal., 2003). Uma das maiores dificuldades, segundo Cohen et al. (2003), na aplicação da WLS é estimar corretamente os pesos a serem utilizados. Para tanto, adotou-se a sugestão de Cohen et al. (2003), descrita a seguir, em que esses pesos são extraídos dos dados.

Primeiro, estima-se a regressão sem empregar a WLS, e os resíduos são salvos para cada observação. Esses resíduos são elevados ao quadrado. Faz-se, então, uma nova regressão em que os resíduos ao quadrado são a VD, e a VI é a mesma da primeira regressão. Salvam-se os valores previstos da VD da segunda regressão para cada objeto, ou seja, os valores previstos dos resíduos ao quadrado. A variável que será utilizada como peso será, portanto, o inverso desses valores previstos. O detalhamento do procedimento pode ser encontrado em Cohen et al. (2003, p. 147). Neste artigo, foi estimada a regressão em que a VD era o desempenho e a VI eram as três diferentes configurações, e seguiramse os passos descritos anteriormente, salvando-se a variável chamada peso, que serviu como fator de ponderação na regressão WLS. Uma das limitações da WLS é que o $\mathrm{R}^{2}$, por conta dos efeitos do peso, não tem o mesmo significado que na OLS ou GLM univariado, sendo assim, não deve ser considerado na análise e interpretação dos dados.

Aplicou-se, então, o teste Levene de igualdade de variância entre os grupos, o qual não apresentou significância estatística, e seguiu-se com o teste do modelo proposto, cujos resultados são apresentados na Tabela 4.

TABELA 4 - Teste do modelo: regressão (WLS) VD - Desempenho

\begin{tabular}{lcccrc}
\hline Variáveis & $\begin{array}{c}\text { Tipo III Soma } \\
\text { dos Quadrados }\end{array}$ & G1 & $\begin{array}{c}\text { Média } \\
\text { Quadrada }\end{array}$ & F & Sig. \\
\hline Modelo Corrigido & $126,542(\mathrm{a})$ & 7 & 18,077 & 18,249 &, 000 \\
Configurações & 98,892 & 2 & 49,446 & 49,915 &, $000^{*}$ \\
Ensino Superior &, 034 & 1 &, 034 &, 035 &, 853 \\
Config*Ens_Sup & 9,866 & 2 & 4,933 & 4,980 &, $009^{*}$ \\
Tempo de Existência da Empresa & 2,952 & 1 & 2,952 & 2,980 &, $087^{\dagger}$ \\
Idade &, 432 & 1 &, 423 &, 427 &, 515 \\
Erro & 107,975 & 109 &, 991 & & \\
Total & 8825,426 & 117 & & & \\
Total corrigido & 234,518 & 116 & & & \\
\hline
\end{tabular}

Nota: a $\mathrm{R} 2=, 540(\mathrm{R} 2$ Ajustado $=, 510)$

b Regressão Mínimos Quadrados Ponderados - Ponderado pela variável Peso

* significativo a $99 \%$

+ significativo a $90 \%$ 
Os resultados corroboram a hipótese testada, isto é, o desempenho é diferente nas três configurações, sendo que a $\mathrm{C} 2$ obteve a maior média de desempenho, seguida da C1 e, com a menor média, a C3. A Tabela 5 apresenta as médias marginais estimadas pelo modelo, levando em consideração os covariantes. A comparação pairwise apresentou diferença significativa a 95\% entre as médias das três configurações. E o teste $\mathrm{F}$ do efeito das configurações apresentou significância estatística a 99\%.

TABELA 5 - Média marginal estimada para o efeito principal no modelo testado

\begin{tabular}{llll}
\hline & C1 & C2 & C3 \\
\hline Desempenho & 3,64 & 4,18 & 3,04 \\
\hline
\end{tabular}

Essa diferença no desempenho é significativa, mesmo com as variáveis de controle Ensino Superior, Tempo de Existência da Empresa e Idade do proprietário presentes no modelo, o que reforça a utilização da taxonomia, já que ela ajuda a explicar a diferença de desempenho entre as organizações. É importante destacar também a significância estatística da interação entre as configurações e a variável Ensino Superior, pois reforça o argumento de que a taxonomia proposta contribui para explicar as diferenças de desempenho, pois isoladamente a formação acadêmica não contribui para a explicação do desempenho, mas associada às configurações ela aparece estatisticamente significativa, com $99 \%$. Entre as outras variáveis de controle, destaca-se o Tempo de Existência da Empresa, com significância estatística de $90 \%$.

\section{DISCUSSÃO DOS RESULTADOS}

Os resultados das análises feitas indicam consistência da taxonomia proposta. As características dos grupos parecem ser distintas o suficiente para que as organizações em cada grupo possam ser consideradas como possuindo diferentes configurações estratégicas, em virtude das diferenças de médias estatisticamente significativas. Soma-se a isso o fato de que as configurações apresentam características únicas que podem ser consideradas distintivas de cada grupo, como, por exemplo, a diferença de média do Locus de Controle Externo entre a C1 e duas outras configurações. Enquanto para esse construto a média de C1 é 3,57, para as outras duas configurações as médias são 1,67 para C2 e 1,98 para C3. Uma diferença considerável. Não só isso, mas a variação das composições entre os três construtos é importante.

Em C1, o Locus Externo de Controle é alto e os outros dois construtos são valores intermediários, inclusive com Comportamento Empreendedor não apresentando diferença estatisticamente significativa de C2.Já em C2, as médias se apresentam em extremos, as mais altas para Comportamento Empreendedor e Capacidades, e a mais baixa para Locus Externo de Controle. Por fim, a C3 apresenta a mais baixa média em Comportamento Empreendedor, e médias intermediárias nos outros dois construtos.

Essa taxonomia também ajuda a explicar a variação na média de desempenho organizacional, como corroborou o modelo testado. Isso reforça sua utilidade para a análise das empresas de pequeno porte em virtude das semelhanças nos imperativos tradicionalmente utilizados. Além disso, como argumenta Miller $(1986,1987)$, os tipos organizacionais não podem ser dissociados das características de seu contexto ambiental, o que pode ser identificado com a significância estatística da interação Configuração e Ensino Superior no modelo testado, servindo de mais um argumento para fortalecer a utilização dessa taxonomia.

É preciso, todavia, aprofundar as pesquisas dessas configurações para empresas de pequeno porte e seus imperativos. Esse é um estudo exploratório, ainda limitado pelo número de empresas e pela amostragem utilizada, o que impossibilita a generalização. A sugestão é que futuras pesquisas apliquem essa taxonomia, incluindo outras variáveis organizacionais e ambientais para o refinamento da proposta, e possibilidade de generalização dos resultados.

\section{CONSIDERAÇÕES FINAIS}

Em relação às limitações do estudo são destacadas algumas observações. As configurações estratégicas com os novos imperativos propostos não incluíram na análise temas tradicionais dessa escola de pensamento, como estratégia, liderança, ambiente e 
estrutura. Mesmo que tenha sido levantada a hipótese de que tais elementos não contribuem significativamente para geração de taxonomias de organizações de pequeno porte, a comparação entre taxonomias desenvolvidas a partir desses diferentes conjuntos de imperativos poderia ser realizada em estudos futuros.

A amostra da pesquisa, em número reduzido de observações, não permitiu que fosse testada a estabilidade das configurações quando houvesse uma divisão inicial das observações e fossem geradas em duas amostras a análise de cluster.

Outro cuidado que deve ser tomado na leitura do trabalho, que não se deve aos procedimentos metodológicos do estudo, mas sim à própria característica da escola da configuração (MINTZBERG; AHLSTRAND; LAMPEL, 1998), é que ela se limita a observar alguns poucos padrões de combinações entre imperativos organizacionais. É preciso ter em mente que essa abordagem caminha rumo à construção de uma teoria de médio alcance e que é oposta à natureza universal pretendida de outras teorias organizacionais. Desse modo, as configurações e seus elementos constituintes são apenas ilustrativos e não exaustivos (MILLER, 1996).

Assim, não se pretende estabelecer, por meio da observação dos resultados desse estudo, que existem apenas três possíveis configurações estratégicas no conjunto de organizações que atua no setor do varejo de vestuário da cidade de Curitiba. Ao contrário, admite-se a possibilidade de outras configurações. Sobretudo, o que se pretendeu analisar foi a possibilidade de identificar uma taxonomia com configurações que possam explicar a variabilidade de desempenho percebida nesses negócios.

Os resultados das análises apontaram a utilidade emidentificar taxonomias ou configurações estratégicas a partir dos novos imperativos propostos para o caso de organizações de pequeno porte. Tal assertiva é reforçada pela corroboração da hipótese de pesquisa que está centrada na lógica da relação entre diferentes configurações e diferentes níveis de desempenho.

\section{REFERÊNCIAS}

ATUAHENE-GIMA, K.; KO, A. An empirical investigation of the effect of market orientation and entrepreneurship orientation alignment on product innovation. Organization Science, v. 12, n. 1, p. 54-74, 2001.
BABBIE, E. R. The practice of social research. 2nd ed. Califórnia: Wadsworth Publishing Company, 1998.

BARDIN, L. Análise de conteúdo. Lisboa: Edições 70, 1977.

BARNEY, J. B. Gaining and sustaining competitive advantage. 2nd ed. Massachusetts: Addison-Wesley Publishing Company, 1996.

BIRKINSHAW, J. Entrepreneurship in multinational corporations: the characteristics of subsidiary initiatives. Strategic Management Journal, v. 18, n. 3, p. 207-229, 1997.

BOYDSTON, M.; HOPPER, L.; WRIGHT, A. Locus of control and entrepreneurs in a small town. 2000. Disponível em: <http://www.sbaer.uca.edu/research/ asbe/2000/23.pdf>. Acesso em: 06 abr. 2007.

BROWN, T. E.; DAVIDSSON, P.; WIKLUND, J. An operationalization of Stevenson's conceptualization of entrepreneurship as opportunity-based firm behavior. Strategic Management Journal, v. 22, n. 10, p. 953968, 2001.

CANEDO, M. T. P.; KRUGLIANSKAS, I. Perfil do empreendedor e conhecimento sobre o cliente como condicionantes do desempenho de supermercados. In: ANGELO, C. F.; SILVEIRA, J. A. G. (Coord.). Varejo competitivo. São Paulo: Atlas, 1999. p. 130-148.

CHAKRAVARTHY, B. S. Measuring strategic performance. Strategic Management Journal, v. 7, n. 5, p. 437-458, 1986.

COHEN, J. et al. Applied multiple regression/correlation analysis for the behavioral sciences. $3 \mathrm{rd} \mathrm{ed}$. Mahwah: LEA Publishers, 2003.

COOPER,A.C.;DUNKELBERG,W.C.Entrepreneurship and paths to business ownership. Strategic Management Journal, v. 7, n. 1, p. 53-68, 1986.

COVIN, J. G.; SLEVIN, D. P. Strategic management of small firms in hostile and benign environments. Strategic Management Journal, v. 10, n. 1, p. 75-87, 1989.

CRAMER, D. Advanced quantitative data analysis. Maidenhead: Open University Press, 2006.

DESS, G. G.; ROBINSON Jr., R. B. Measuring organizational performance in the absence of objective measures: the case of the privately-held firm and conglomerate business unit. Strategic Management Journal, v. 5, n. 3, p. 265-275, 1984. 
FISS, P. C. A set-theoretic approach to organizational configurations. Academy of Management Review, v. 32, n. 4, p. 1180-1198, 2007.

GALUNIC, D. C.; RODAN, S. Resource recombinations in the firm: knowledge structures and the potential for Schumpeterian innovation. Strategic Management Journal, v. 19, n. 12, p. 1193-1201, 1998.

GIMENEZ, F. A. P. O estrategista na pequena empresa. Maringá: Edição do autor, 2000.

HAIR Jr., J. F. et al. Multivariate data analysis with readings. 4th ed. New Jersey: Prentice-Hall, 1995.

JACOBSON, R. The "Autrian" school of strategy. Academy of Management Review, v. 17, n. 4, p. 782807, 1992.

JARZABKOWSKI, P. Strategy as practice: recursiveness, adaptation and practices-in-use. Organization Studies, v. 25 , n. 4 , p. 529-560, 2004.

KAUFMANN, P.; WELSH, D.; BUSHMARIN, N. V. Locus of control and entrepreneurship in the Russian Republic. Entrepreneurship Theory and Practice, v. 20, n. 1, p. 43-56, 1995.

KING, A. W.; ZEITHAML, C. P. Competencies and firm performance: examining the causal ambiguity paradox. Strategic Management Journal, v. 22, n. 1, p. 75-99, 2001.

LEONARD-BARTON, D. Core capabilities and core rigidities: a paradox in managing new product development. Strategic Management Journal, v. 13, p. 111-125, 1992.

LOOSEMORE, M.; LAM, A. S. Y. The locus of control: a determinant of opportunistic behavior in construction health and safety. Construction Management and Economics, v. 22, p. 385-394, 2004.

McGRATH, R. G. Entrepreneurship, small firms and wealth creation: a framework using real options reasoning. In: PETTIGREW, A.; THOMAS, H.; WHITTINGTON, R. (Ed.) Handbook of strategy and management. London: Sage, 2002. p. 299-325.

MENNA, H. L.; ROSSI, C. A. V. Orientação para o mercado e performance: evidências em empresas gaúchas de varejo de confecções masculinas. In: ANGELO, C. F.; SILVEIRA, J. A. G. (Coord.). Varejo competitivo. São Paulo: Atlas, 2001. p. 154-177.
MILLER, D. Configurations of strategy and structure: towards a synthesis. Strategic Management Journal, v. 7, n. 3, p. 233-249, 1986.

The genesis of configuration. Academy of Management Review, v. 12, n. 4, p. 686-701, 1987.

Configurations revisited. Strategic Management Journal, New York, v. 17, n. 7, p. 505512, 1996.

MILLER, D.; FRIESEN, P. H. Archetypes of strategy formulation. Management Science, v. 24, n. 9, p. 921 933, 1978.

MILES, R. E.; SNOW, C. C. Organizational strategy, structure and process. New York: McGraw-Hill, 1978.

MINTZBERG, $H$. The structuring of organizations. New Jersey: Prentice Hall, 1979.

MINTZBERG, H.; AHLSTRAND, B.; LAMPEL, J. Strategy safari. New York: The Free Press, 1998.

MUELLER, S. L.; THOMAS, A. S. Culture and entrepreneurial potential: a nine country study of locus of control and innovativeness. Journal of Business Venturing, v. 16, n. 1, 2001.

NAMAN, J. L.; SLEVIN, D. P. Entrepreneurship and the concept of fit: a model and empirical tests. Strategic Management Journal, v. 14, p. 137-153, 1993.

PEDHAZUR, E.; SCHMELKIN, L. Measurement, design and analysis. Hillsdale: LEA Publisher, 1991.

PORTER, M. E. Competitive strategy. New York: Free Press, 1980.

PUGH, D. S.; HICKSON, D. J.; HININGS, C. R. An empirical taxonomy of structures of work organizations, Administrative Science Quarterly, v. 14, n. 1, p. 115126, 1969.

RUGMAN, A. M.; VERBEKE, A. Edith Penrose's contribution to the resource-based view of strategic management. Strategic Management Journal, v. 23, p. 769-780, 2002.

SANCHEZ, R. Analyzing internal and competitor competences: resources, capabilities, and management processes. In: FAULKNER, D. O.; CAMPBELL, A. (Ed.). The Oxford handbook of strategy. Oxford: Oxford University Press, 2003. p. 344-371. 
SHANE, S.; VENKATARAMAN, S. The promise of entrepreneurship as a field of research. Academy of Management Review, v. 25, n. 1, p. 217-226, 2000.

SHARMA, P.; CHRISMAN, J. J. Toward a reconciliation of the definitional issues in the field of corporate entrepreneurship. Entrepreneurship Theory and Practice, v. 23, n. 3, p. 11-27, 1999.

SNOW, C. C.; MILES, R. E.; MILES, G. A configurational approach to the integration of strategy and organization research. Strategic Organization, v. 3, n. 4, p. 431-439, 2005.

STEVENSON, H. H.; JARILLO, J. C. A paradigm of entrepreneurship: entrepreneurial management. Strategic Management Journal, v. 11, p. 17-27, 1990. Special Issue.

VENKATRAMAN, N.; PRESCOTT, J. E. Enviromentstrategy coalignment: an empirical test of its performance implications. Strategic Management Journal, v. 11, n. 1, p. 1-23, 1990.

Recebido: 25/09/2009

Received: 09/25/2009

Aprovado: 11/12/2009

Approved: 12/11/2009 